\title{
Pediatric oncology and the future of oncological imaging
}

\author{
Stephan D. Voss
}

Received: 10 January 2011 / Accepted: 20 January 2011

(C) Springer-Verlag 2011

\begin{abstract}
The future of pediatric oncology will be influenced by changes in drug design and treatment strategy, with genomic medicine and molecular-based diagnostics and therapeutics playing increasingly important roles. The role of imaging as a means of measuring response to therapy has also evolved, with the development of new technologies and higher sensitivity means of detecting tumors. Conventional anatomical imaging techniques are being increasingly supplemented with functional techniques, including FDG-PET imaging and diffusionweighted MR imaging. The risk-adapted treatment regimens of the past, which led to improved event-free and overall survival in many pediatric cancers, have paved the way for new response-based treatment paradigms. Response-based approaches seek to identify patients with a high likelihood of cure, treating them less aggressively, while those not responding to therapy are identified early and redirected into more aggressive therapeutic regimens. These advances will require concurrent development of imaging biomarkers as surrogates of early response to therapy. Incorporating these techniques into new responsedirected treatment algorithms will be crucial as personalized medicine and molecular-targeted, tumor-specific therapies gain acceptance for the treatment of children with cancer.
\end{abstract}

Disclaimer The supplement this article is part of is not sponsored by the industry. Dr. Voss is supported by NIH/NCI grants U10 CA098543, UO1 CA097452, NIH grant UL1 RR02575.

S. D. Voss $(\square)$

Department of Radiology, Children's Hospital Boston,

300 Longwood Ave.,

Boston, MA 02115, USA

e-mail: stephan.voss@childrens.harvard.edu
Keywords Pediatric cancer - Molecular imaging · Diffusion imaging $\cdot$ Response assessment

\section{Introduction}

During the last decade, a number of promising technical advances were made in radiology. PET-CT was introduced in the late 1990s and its use has now become nearly universal for diagnosing, staging and evaluating response in patients with cancer. In addition, the concept of molecular imaging was introduced with the development of contrast agents that target tissues and activate enzymes. Optical imaging emerged as a novel means of imaging tumors, with use of near-infrared fluorescent imaging, bioluminescent imaging and quantum dots. With these new developments came the great promise for molecular imaging to improve cancer detection and aid in treatment assessment. However, a decade later, most of these agents have yet to be introduced into the clinical arena and there are still many challenges that remain in pediatric oncology and oncological imaging. Foremost among these challenges will be how we use the technological advances to improve the assessment of patients with cancer. Developing objective measures of treatment response is critical in order to have evaluable prospective end points in early-phase clinical trials and to determine whether new agents warrant further testing. The challenge is developing a surrogate end point that accurately reflects the disease process and response to therapy at a time when other indicators of response (e.g., change in clinical status) may not reflect treatment response. This is critical as we move from a treatment paradigm that has been primarily risk-adapted (i.e. low-, intermediate- and high-risk patients) to response-based treatment regimens (i.e. treatment stratification based on early response to therapy, rather than pretreatment risk classification). 
As early as 1960, oncologists were concerned with accurately determining treatment response and "treatment was considered to give a positive response if either the total measured tumor mass decreased with no lesions increasing in size and no new lesions appearing or if the group of treating physicians considered that the treatment had been of benefit to the patient as a whole" [1]. This early definition allowed for subjective assessments of patient benefit to override the few objective response criteria that were available. Since that time, much effort has been directed at developing and optimizing a more objective set of criteria based on imaging findings. Putting these criteria to the test in formal clinical trials will be the main challenge for the next decade. We should no longer be relying on uni- or bidirectional changes in tumor size as the sole means of determining treatment response.

We are fortunate that there is a relatively high rate of survival in children with cancer. However, this was largely the result of treatment advances made during the past two decades; since then, there have been relatively modest gains in developing new treatment approaches (Fig. 1). Those patients who continue to have resistant disease represent some of the most challenging patients to treat, which has led to many innovative new treatment strategies. These new approaches to treatment and drug design, in turn, affect how we assess response to therapy [2-4]. Newer molecular-based therapies often do not produce the cytolytic effects typical of conventional chemo- therapeutic agents. Such targeted therapies may have an effect on a particular metabolic pathway and the imaging techniques chosen to assess response to such targeted agents will require strategies that evaluate the changes in the metabolic pathway being targeted, i.e. tyrosine kinase inhibition or inhibition of DNA synthesis, both downstream effects that can result from targeting a particular molecular pathway. Without a thoughtful approach to imaging, subtle but very real therapeutic responses may go unnoticed.

How should we go about approaching these many new challenges? A systematic approach will be needed. Initial efforts to develop objective measurement criteria for assessing solid tumors were put forward by the World Health Organization (WHO) and utilized bidirectional measurement techniques [5]. Treatment was largely risk-adapted and these measurement techniques focused primarily on disease staging and on determining initial disease bulk in an effort to stratify patients into treatment groups. Early criteria for response were also developed and led to categories ranging from complete response to progressive disease. The criteria also included stable/no change or partial response in the classification scheme, depending on the estimates of change in tumor size. The WHO criteria presented many challenges, particularly for tumors such as Hodgkin lymphoma, bone and soft tissue sarcoma, and neuroblastoma (where both measurable disease and infiltrative marrow disease may be present). Minimum lesion size and number of lesions to be
Fig. 1 Overall Survival Data from Children's Oncology Group cooperative studies shows only modest gains in survival for the majority of pediatric malignancies since the 1990s, emphasizing the need for novel approaches to treating pediatric cancer (Courtesy of Dr. Greg Reaman, group chairman, Children's Oncology Group)

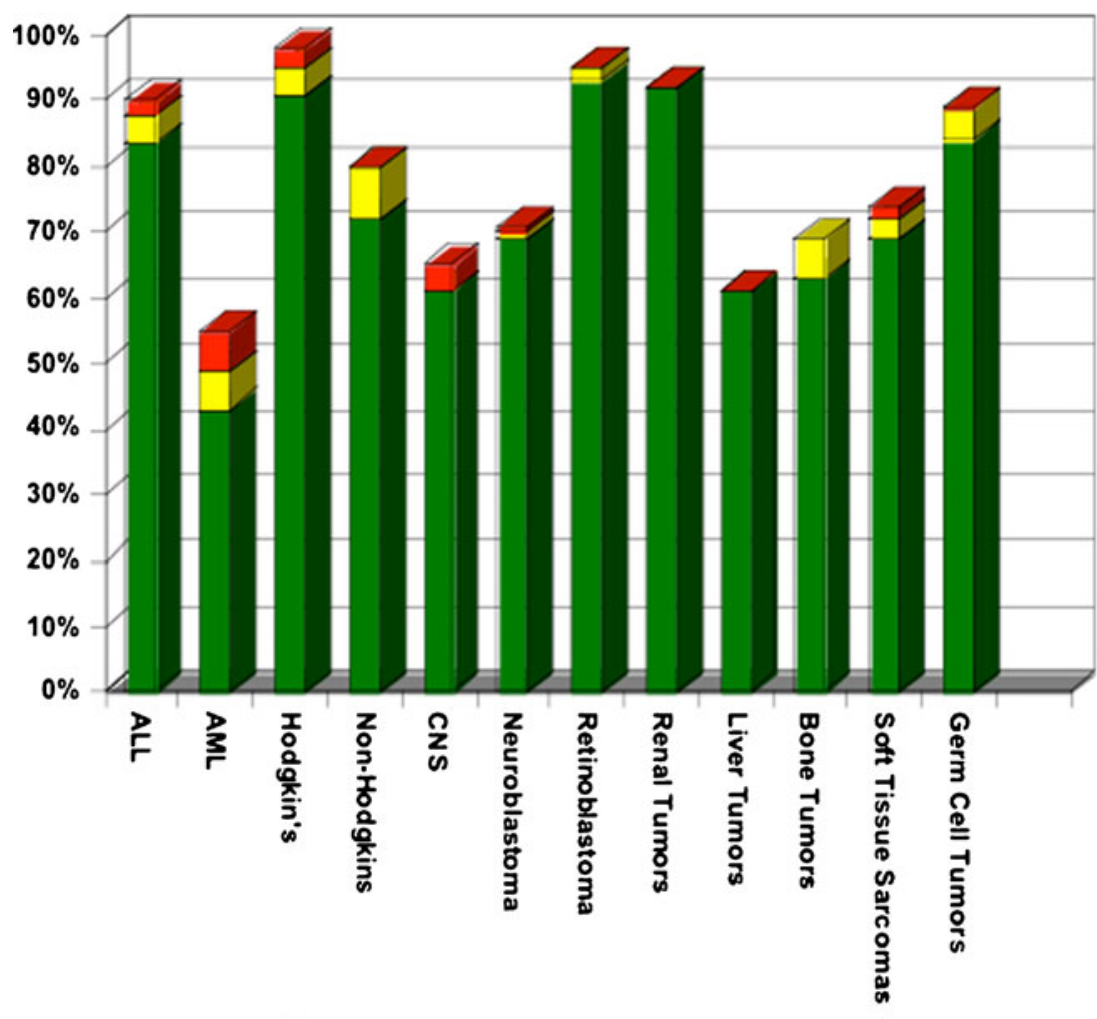

2000-2005 $\square$ 1995-2000 $\square 1990$ 
recorded were not specified and depending on the location of tumor, size measurements may have been based on physical exam estimates. The WHO criteria also did not account for tumors like Hodgkin lymphoma and the sarcomas, which frequently leave residual post-treatment scar tissue. In addition, these early methods were devised before the advent of multiplanar cross-sectional imaging techniques in common use today (CT and MRI). Also, the choice of measurement dimension often led to errors in characterizing disease progression and disease response, depending on which dimension of the tumor changed in size.

\section{Changes in therapy: nanomedicine and the emergence of personalized medicine}

As we revise our approach to assessing response to therapy we must first educate ourselves on the newer treatment techniques, the agents being developed and the physiological processes being targeted. The concept of nanomedicine has recently emerged $[6,7]$. This field has been developing parallel to the new developments in imaging during the past two decades. For example, the use of nanoparticles for tumor detection has recently received FDA approval for certain tumors. Historically, the earliest nanoparticles were antibody-based. More recently, elegant boutique nanoparticles have been developed and are being used to either directly diagnose or treat tumors or, alternatively, to aid in the transport of diagnostic or therapeutic agents through biological barriers, such as cell membranes, to gain access to intracellular molecules or to mediate intermolecular interactions [8]. Local accumulation or delivery of nanoparticles can simultaneously be used to detect molecular changes within the adjacent microenvironment. Such nanoparticles can now be engineered to have unique sizes, chemical compositions and physical characteristics to specifically target selected tissues or body compartments.

FDA-approved nanoparticles include liposomes, dendrimers and gold nanoparticles [6]. A systematic review of these new treatment developments is beyond the scope of this summary; however, it has been emphasized that incorporating advanced imaging techniques into the drug development process is essential to ensure that the most promising drug candidates ultimately reach the market $[9,10]$. For example, we know that the average time from synthesis of a new drug to FDA approval is approximately 14 years. Roughly half of this time is spent in Phase I-Phase III clinical trials. It is essential that our imaging techniques are directed toward assessing pharmacological activity and therapeutic efficacy to ensure that the best compounds advance further in the drug development pipeline.

The emergence of personalized medicine in oncology has begun to revolutionize the approach to screening, diagnosing and treating patients with cancer [11]. Cancer screening programs are now offered based on specific genetic detection techniques. Whole genome screening has become a routine exercise in many large cancer centers. Increasingly, patients are being screened and identified as carriers of cancer susceptibility genes. However, as imagers, we will also be faced with how we approach screening this increasing population of asymptomatic patients who are at increased risk of developing cancer. One focus has been on identifying tumor biomarkers. Defining populations of patients based on expression of particular biomarkers and targeting treatment approaches toward specific biomarkers will produce subsets of patients who will require a unique approach to imaging.

Leroy Hood, one of the founders of the revolution in bioengineering and biotechnology development, and coworkers have introduced the concept of P4 Medicine, which emphasizes a merging of technological innovation with personalized care [12]. In particular, they stress that predictive genomics and molecular biomarkers will be essential in alerting patients to the risk of disease before it develops. A personalized approach will then be needed to have treatment and diagnosis tailored to the patient's own molecular biomarker profile in an effort to develop precise and effective therapies that could potentially be administered in a vaccine-like manner to prevent disease before it occurs. This concept, although somewhat futuristic, emphasizes the importance of educating ourselves about new treatment developments and the need for our imaging strategies to be closely aligned with these new approaches.

\section{Putting innovation to the test: moving beyond RECIST}

Response Evaluation Criteria in Solid Tumors (RECIST) was developed both to simplify the measurement process and to increase/improve the accuracy of measurement techniques in predicating response [13]. As with the earlier bidirectional measurement techniques, RECIST still relies on tumor size changes, but measured in a single dimension and requiring measurement of as many as ten lesions in the same patient. The RECIST technique depends on the tumor being a spherical or ellipsoid mass, in which the logarithm of the number of tumor cells present in the mass can be linearly related to the logarithms of diameter, area and volume. With perfectly spherical or ellipsoid masses, diameter, area and volume are all equally valid surrogates of tumor measurement. However, in many pediatric tumors there is considerable heterogeneity in tumor size. As a result, there has been debate on whether to employ bidirectional versus unidimensional measurement techniques and whether these time-consuming techniques are relevant in assessing drug activity and clinical response [14, 15]. Newer 
functional approaches, such as FDG-PET, have only just begun to be incorporated into the routine diagnosis, staging and post-treatment evaluation for some pediatric solid tumors. It is these newer functional techniques that will likely form the basis for pediatric oncological imaging during the next decade [16].

\section{Major technical innovations}

A systematic review of all of the innovations that have occurred and will influence how we image pediatric oncology patients in the decade to come are beyond the scope of this review. Three major areas of innovation will be reviewed in the context of diagnosis and staging, posttreatment response assessment and surveillance imaging:

\section{- ${ }^{18}$ F-FDG-PET imaging}

- Advanced MR imaging (whole-body MRI, diffusionweighted MRI [DW-MRI] and dynamic contrastenhanced MRI [DCE-MRI])

- Fluorescent imaging.

\section{${ }^{18}$ F-FDG-PET imaging}

The discovery that ${ }^{18}$ F-FDG accumulates in metabolically active tumor cells has revolutionized oncological imaging, particularly for those therapeutic agents that inhibit tumor metabolism and proliferation, without producing immediate effects on tumor size. Although this discovery was made several decades ago, the potential for FDG-PET imaging to detect active versus stable disease and to provide more clinical information than simply anatomical localization of disease has just begun to be realized. Newer generation PET scanners are faster and afford higher resolution. FDG-PET has the potential to reflect both the metabolic state and the proliferative potential of residual disease in patients who, after receiving both conventional and experimental therapies, may still have measurable disease by conventional imaging techniques.

There have been a number of studies showing the feasibility of using FDG-PET in the majority of pediatric malignancies [17-20] and our ability to characterize tumors both metabolically and anatomically sets FDG-PET apart from conventional anatomical imaging techniques such as CT, MRI and ultrasound. Metabolic changes induced by chemotherapy often precede morphological changes. Because ${ }^{18}$ F-FDG uptake provides a direct measure of tumor glucose metabolism, FDG-PET evaluation of tumor response to therapy can frequently be seen early after the initiation of treatment, prior to tumor shrinkage (Fig. 2), and in many cases has been shown to predict response to therapy more accurately than conventional modalities. For example, in pediatric Hodgkin lymphoma, Ewing sarcoma and osteosarcoma, studies of small numbers of patients have provided compelling evidence that early decreases in ${ }^{18} \mathrm{~F}$-FDG uptake may identify patients who will fare better than those with residual uptake on PET scans [21-24]. It is attractive to hypothesize that such an "in vivo chemo-sensitivity test" will be important in developing response-based approaches to therapy. However, during the next decade, this hypothesis must be directly tested and expanded to include a larger number of pediatric tumors. The technology is no longer a limitation - how and where we test the technology is our present challenge, and it remains to be determined whether the best measure of successful tumor treatment will be ultimate tumor shrinkage, the cessation of metabolic activity within the tumor or some combination of factors.

PET has become nearly synonymous with PET imaging using ${ }^{18}$ F-FDG; however, there are many other PET imaging agents in development [25]. These include radiolabeled small molecules such as neurotransmitters, peptides, antibodies and antibody derivatives. Virtually any molecule that can be chemically modified to incorporate a PET isotope can be used for PET imaging. Relative to other nuclear medicine techniques, PET imaging has the advantage of high sensitivity and an inherently greater image resolution. While ${ }^{18} \mathrm{~F}$-fluorine is the most commonly utilized positron emitting radionuclide, other PET isotopes such as ${ }^{124} \mathrm{I},{ }^{11} \mathrm{C}$ and ${ }^{64} \mathrm{Cu}$ are also being developed for clinical use [25]. ${ }^{64} \mathrm{Cu}$, in particular, has emerged as one of the most promising PET tracers for both diagnosis and - together with ${ }^{67} \mathrm{Cu}$ - treatment of cancer. With a half-life of $12.7 \mathrm{~h},{ }^{64} \mathrm{Cu}$ is appropriate for radiolabeling antibodies and peptides and can be used for PET imaging both for diagnosis and for determining dosimetry prior to radio-conjugate immunotherapy [26].

${ }^{18}$ F-fluoro-thymidine (FLT) is also being evaluated in clinical trials. Thymidine is a precursor for DNA synthesis and ${ }^{18} \mathrm{~F}$-fluoro-thymidine, like the ${ }^{3} \mathrm{H}$-thymidine analogue that has been used for years in molecular biology laboratories, can be used to directly measure DNA synthesis [27]. Incorporation of ${ }^{18} \mathrm{~F}$-FLT into tumor DNA allows assessment of DNA synthesis and is a direct means of assessing tumor proliferative activity. In some instances, particularly in the brain, where high levels of background metabolic activity and glucose uptake are present, the use of FLT may be an effective alternative to assessing treatment response. However, as was emphasized earlier, the choice of imaging technique must be based on the therapy being tested. If the therapeutic agent being evaluated is not expected to have a direct effect on DNA synthesis, the use of ${ }^{18}$ F-FLT would not be a suitable choice for evaluating drug activity.

There is little data on the utility of ${ }^{18}$ F-FLT in pediatric tumors, although during the next decade it is expected that this agent will be used with increasing frequency. Indeed, 
Fig. 2 MRI and PET images from a patient with Ewing sarcoma being treated on a phase I COG study evaluating safety and efficacy of IGFR-targeted therapy. The upper row of images shows a large soft-tissue mass with intense FDG uptake at baseline. After 1 month of IGFRtargeted therapy, the size of the tumor has not significantly changed, but the intensity of FDG uptake has decreased to nearly background levels, reflecting significant treatment-induced reduction in tumor metabolic activity (Presented at ASCO 2009 Annual meeting [28])

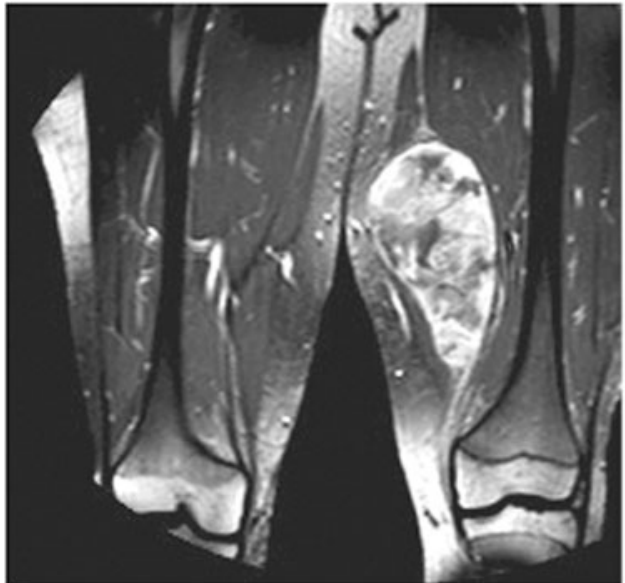

Post-Gd T1 MRI

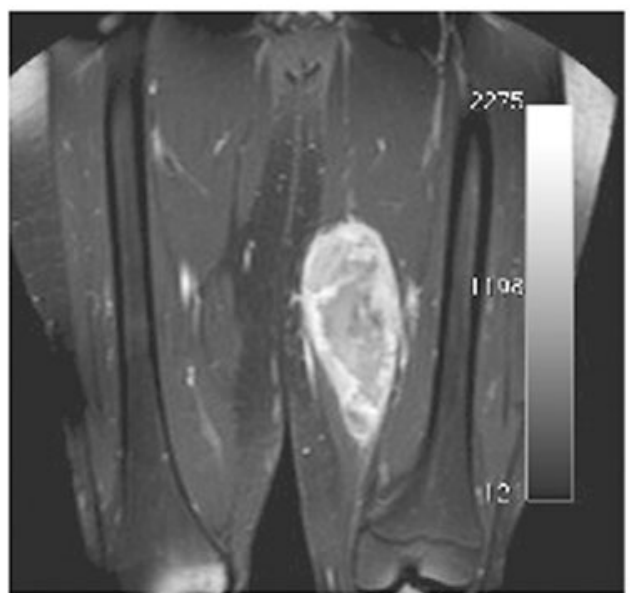

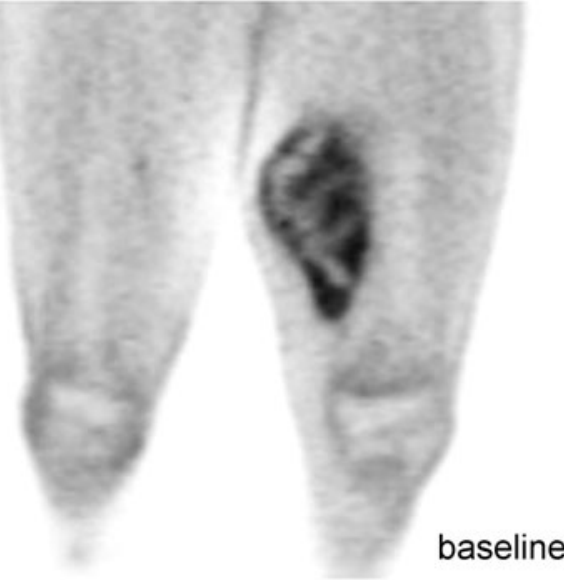

PET

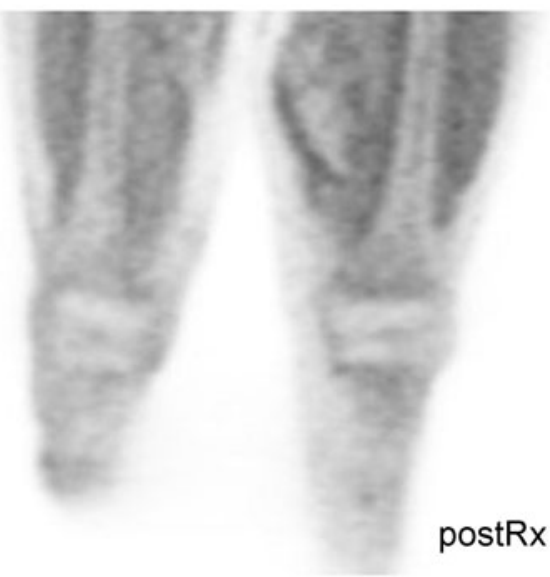

for the purpose of specifically investigating anti-proliferative agents and detecting residual neoplastic tissue with a high proliferation rate, FLT may be more cancer specific than FDG. Similarly, there are many therapies that rely on the induction of apoptosis, as opposed to direct cytolysis. Imaging agents that directly measure apoptosis by recognizing phosphatidylserine residues exposed on the apoptotic cells have been shown in early phase trials to identify populations of patients who have responded to chemotherapy, distinguishing them from those who have progressive disease [29].

\section{MRI-PET}

PET imaging in most centers is now performed on integrated PET-CT scanners. In the coming decade, the evolution of PET-MRI for routine body imaging is likely to completely revolutionize our approach to imaging patients with cancer [30, 31]. The idea of combining PET and MRI is not new, and was discussed as early as the mid-1990s, even before the introduction of PET-CT. PET imaging always requires some surrogate technique for soft-tissue attenuation correction. The use of CT was obvious since Houndsfield unit intensity measurements directly correlate with soft-tissue attenuation of the x-ray beam. Furthermore, it was shown in multiple studies that the soft-tissue attenuation predicted by $\mathrm{CT}$ Houndsfield unit measurements could be used to effectively correct the soft-tissue attenuation effects on the $511 \mathrm{keV}$ photon emitted from PET isotopes. As a result, integrated PET-CT scanners were developed and nearly all PET imaging today is performed with integrated PET-CT systems. However, the growing concern over radiation exposure related to diagnostic imaging in oncology patients [32] has led some to re-evaluate CT doses needed for the purpose of attenuation correction [33], and furthermore to question the need for diagnostic-quality CT scans during PET CT. It is clearly essential to have some anatomical cross-sectional imaging technique with which to correlate functional imaging findings. The integrated PETCT provides direct anatomical landmarks with which to correlate areas of abnormal FDG uptake and has been shown in multiple studies to increase the sensitivity and specificity of both individual PET and CT techniques.

Independent of these dose-reduction strategies in PET $\mathrm{CT}$, during the past 5 years integrated PET MRI scanners have been developed and have now reached the clinical setting [31, 34]. It is well appreciated that MRI provides 
superior characterization of soft-tissue abnormalities. MRI can be used to derive metabolic and functional activity about tissues, and the advantages to developing integrated PET-MRI systems seem obvious. The technical challenge has been relating the MR image information back to the PET data to accomplish accurate attenuation correction. This challenge is not insignificant, since the signal intensities and the MR properties of specific tissues relate to inherent $\mathrm{T} 1$ and $\mathrm{T} 2$ properties of the tissues, but are also influenced by magnetic field strength, choice of pulse sequence, presence or absence of gradients, choice of radiofrequency coil and often lengthy acquisition times, resulting in motion artifact. Furthermore, the incorporation of PET detectors into high field strength magnets presents a further challenge. Despite this, the development of PETMRI scanners has the potential to completely change our approach to clinical molecular imaging since the anatomical and functional information derived from both modalities is likely to be synergistic [35]. For pediatric patients, the enthusiasm around PET-MRI is justified, since no additional $\mathrm{CT}$ radiation is required and integrated systems should allow both PET and MRI scanning to be done simultaneously, reducing the need for prolonged sedation in our very young patients. This has only been possible with the development of recent faster MR imaging techniques, which allow whole-body examinations to be performed using multiple receiver coils and parallel imaging techniques. The incorporation of PET-MRI into the pediatric imaging armamentarium will be exciting, but challenging. We will need to validate this new technology and demonstrate that this new technique addresses clinical needs by improving diagnostic accuracy, treatment management and ultimately cost efficiency.

\section{MRI}

\section{Diffusion-weighted MR imaging}

Diffusion-weighted MR imaging (DWI-MRI) has been used clinically for many years, primarily in neuroradiology where the use of diffusion imaging dramatically changed our ability to image cerebral ischemia and identify patients who would be amenable to early treatment intervention with thrombolytics and anticoagulents. With the development of faster scanning techniques, it has now become possible to routinely perform DWI in the abdomen. DWMRI has emerged as a potential novel biomarker in oncological imaging, where it has been linked to lesion aggressiveness and tumor response [36, 37]. A recent report from the National Cancer Institute emphasized the importance of DW-MRI as a cancer biomarker and provided a consensus panel's recommendations for how to validate and incorporate DWI into oncological imaging protocols [36].
DW-MRI relies on imaging the microscopic mobility of water. This mobility, known as Brownian motion, is highly influenced by the microenvironment around the water. For example, impaired water movement in and out of cells due to ischemia manifests as restricted diffusion. Similarly, highly ordered tissues are expected to have more restricted free water diffusivity as compared to free fluid, or to tissues with limited or no cellularity. As with most tissues examined using MR imaging, individual diffusion measurements for individual tissues differ [38]. However, in general, it has been recognized that cellular tumors typically manifest restricted diffusivity to a degree greater than the surrounding tissues, allowing for increased lesion conspicuity. DW-MRI has the advantage of not requiring intravenous contrast agents, and small cellular lesions can be detected with exquisite sensitivity using DW-MRI, often when they are below the limits of detection with other techniques (Fig. 3). Furthermore, changes in the tumor in response to therapy, such as tumor necrosis, can be expected to decrease cellular density and increase intraand extracellular free water, resulting in changes in diffusivity in response to therapy. This underscores the two main uses for which DWI in oncology has been incorporated: improved lesion detection and assessment of response to therapy.

As a biomarker for the quantitative evaluation of tumor response to therapy, DWI is especially appealing as a means of quantifying the diffusivity of water molecules within a tumor. The Apparent Diffusion Coefficient (ADC) map is used to measure the actual diffusivity of the tissue. Changes in the ADC values are inversely correlated with tumor cellularity and are used to evaluate the tumor's response to therapy [38]. Current techniques use the mean or median ADC value of an ROI within the tumor to quantify the tumor response. However, we have found the response of $\mathrm{ADC}$ to therapy in the clinical setting to be more complex to quantify due to inherent pretreatment and post-treatment heterogeneity observed within human tumors (Fig. 4) and use of ADC response will require development of advanced measurements to quantify the tumor response throughout the entire tumor volume with greater accuracy. It must be said that the physical basis for why malignant tumors have lower ADC values is not completely understood, but probably relates to a combination of inherent increased cellularity and tissue disorganization, resulting in reduced motion of free water within the tumor [36]. More work will be needed during the coming years to determine which tumors can be expected to show restricted diffusion on routine imaging sequences. Furthermore, how tumors will respond to therapy and how these changes in diffusivity are reflected in patient outcome remain to be determined for the majority of pediatric solid tumors. 
Fig. 3 Fat-suppressed T2-W MRI, DW-MRI, CT and fused $\mathrm{PET} / \mathrm{CT}$ images in a patient with adrenal cortical carcinoma show intense FDG uptake and restricted diffusivity in the large metastatic liver lesions in the right liver lobe. The tiny lesion in the left lateral segment of the liver (arrow) is clearly detectable on the diffusionweighted MR images (DWI), but is inconspicuous or undetectable with the other images sequences and techniques, emphasizing the role that DW-MRI plays in improving sensitivity for lesion detection
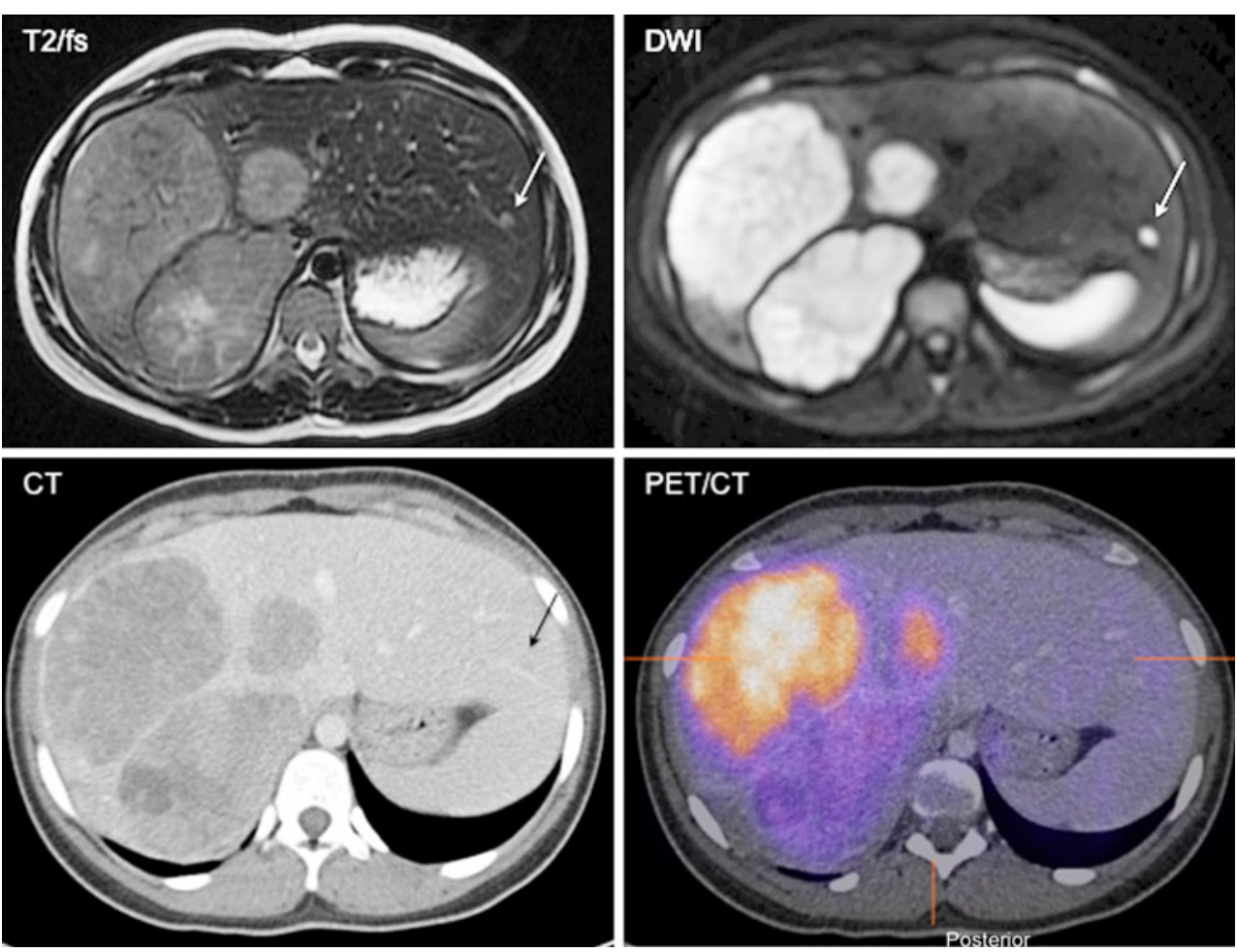

Equally important is the potential ability for DWI-MRI to distinguish between benign and malignant lesions. There are several studies showing that the apparent diffusion constant (a calculated value which reflects actual tumor diffusivity, accounting for background signal due to inherent T2 properties of the tissue and blood flow/ perfusion effects) can be used to help distinguish between benign and malignant lesions [38]. As shown in Fig. 3, DWI can afford exquisitely sensitive lesion detection and increased conspicuity of lesions that aren't otherwise apparent on both conventional T2 and post-gadolinium enhanced MR images. Furthermore, changes in tumor diffusivity can be seen even when tumor shrinkage has not yet occurred. It has been suggested that DW-MRI may
Fig. 4 Diffusion-weighted $(b=400)$ and fat-suppressed T2-W MR images are shown together with the calculated ADC map in a patient with neuroblastoma. The left pedicle lesion in the vertebral body is easily seen on the DW-MR images (arrow), as well as the patient's primary tumor. The ADC map confirms restricted diffusivity throughout the tumor. The histograms demonstrate the composite of ADC values calculated throughout the entire tumor volume and emphasize both the heterogeneity of the tumor ADC values, and value of such an analysis in measuring quantitative changes in the tumor during therapy: compare the preand post- histograms, showing a global increase in ADC following therapy, reflecting an increase in diffusivity, possibly related to tumor necrosis, decreased tumor cellularity or both
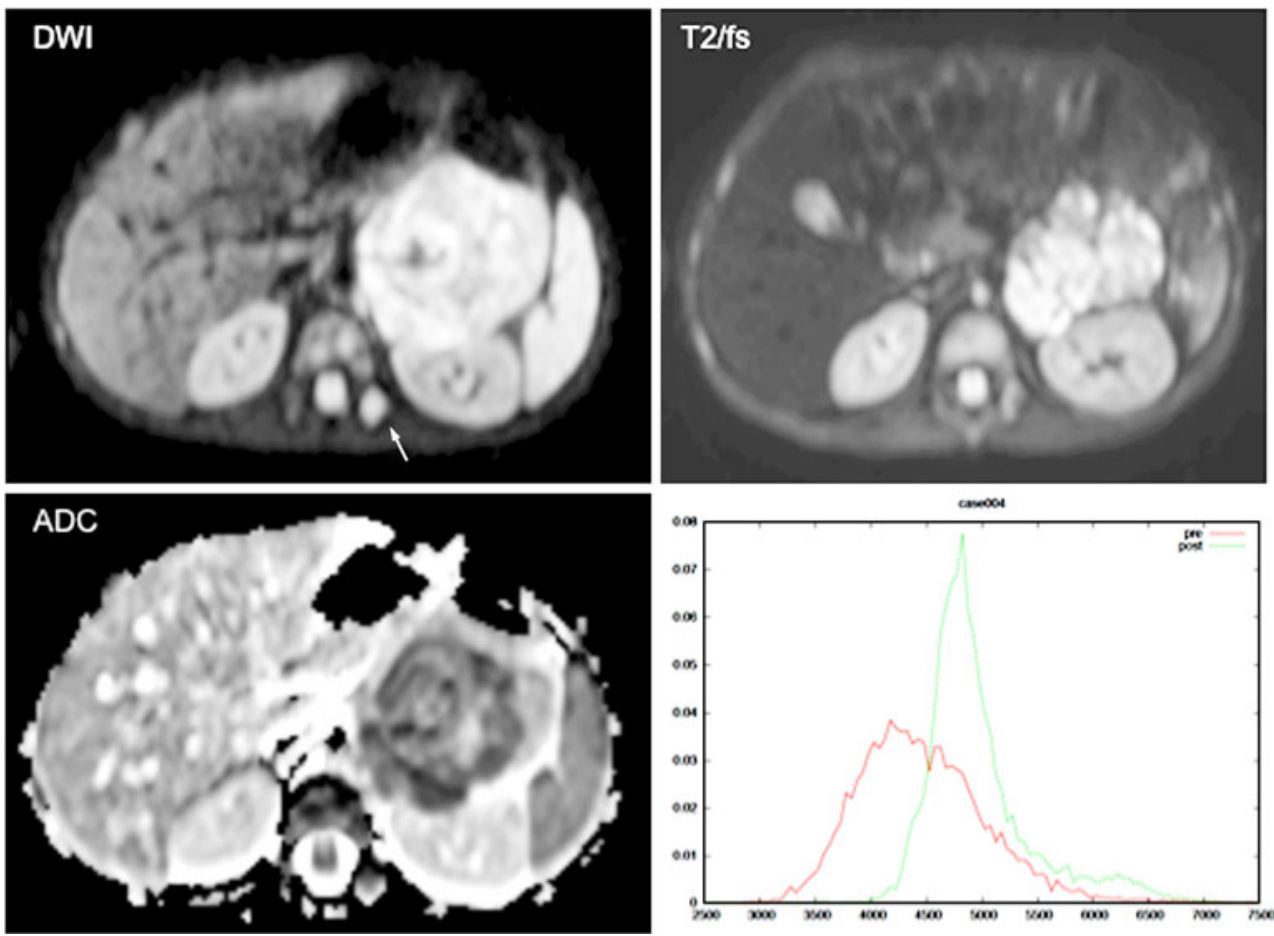
be an effective early biomarker for treatment outcome for drugs that disrupt the vasculature and induce apoptosis, since cellular death and vascular changes, which can be reflected in changes in diffusivity, frequently precede changes in lesion size [36]. Preclinical work has shown that DWI can distinguish between nonperfused viable and nonperfused necrotic tissue. In several early clinical trials, rising ADC values (reflecting increasing diffusivity within the tumor, presumably related to necrosis) were shown in response to therapy in both primary and metastatic tumors. A corollary to this is the absence of a significant change in tumor size or an increase in size coupled with a persistently low ADC (highly restricted diffusivity) may add additional evidence supporting a lack of tumor response to therapy. It is likely that changes in ADC will also reflect tissue dehydration and fibrosis, in addition to tumor necrosis, and the resultant overall change in ADC will probably be a complex mix of biophysical processes. This will require a systematic approach to assessing and correlating ADC values with tissue biopsy when possible and with ultimate patient outcome. This correlation will determine whether monitoring changes in diffusivity will be a reasonable correlate for response and whether it indeed has prognostic significance in distinguishing satisfactory to poor patient outcome [39].

There have been relatively few studies assessing DWI in pediatric solid tumors. One study which evaluated 19 non-CNS tumors, including neuroblastoma, Wilms tumor, rhabdomyosarcoma and a variety of other pediatric solid tumors, showed a significant relationship between cellularity and ADC. However, these authors emphasized that the use of the $\mathrm{ADC}$ value alone could not be used to differentiate benign from malignant lesions [40]. Overall, therefore, DWI-MRI is likely to play a role in tumor staging, identifying sites of disease that would otherwise be radiographically occult and potentially assessing response as a predictive parameter for treatment outcome. The ability to distinguish active from residual/relapse disease and whether the use of DWI will be an effective biomarker for molecular-based therapies remain to be determined.

\section{Whole-body MRI}

The emergence of higher field strength magnets, faster imaging sequences and whole-body phased array coils, coupled with dynamic table movement capabilities within the magnet, have made whole-body imaging techniques for children increasingly available. With increasing concern about radiation dose, the ability for whole-body MRI to allow a single whole-body evaluation for malignant disease to be obtained without additional radiation exposure is of particular interest [41-44]. In the past, whole-body MRI in children has been largely used to evaluate bone marrow disease. However, a recent study, reviewing results of 24 children with a variety of pediatric solid tumors, showed high sensitivity for detection of malignant disease [43]. By combining whole-body MRI techniques for routine tumor surveillance with DW-MRI and potentially whole-body diffusion techniques for both lesion characterization and enhanced lesion detection, whole-body imaging may soon be used as the primary means of assessing the majority of pediatric solid tumors.

Major limitations have been respiratory and cardiac motion and the long duration of typical whole-body scans. However, the use of parallel imaging techniques, respiratory triggering and cardiac gating has effectively eliminated motion-related artifacts. Faster scanning sequences and multiple coherent body coils typically allow the entire body to be imaged in two or three table positions/fields of view. As with any comprehensive examination, there is some expected loss of resolution and tiny sub-centimeter lesions may go undetected. However, in studies comparing whole-body imaging techniques to other tumor staging modalities, whole-body MRI and FDG-PET have been, in most instances, comparable and, in many instances, complementary [43].

As discussed earlier, an obvious future use of wholebody MRI, together with diffusion imaging, will be the integration of whole-body MRI together with PET in our new generation MRI-PET scanners. Suddenly we have the ability to perform a sensitive, high-quality, anatomical imaging technique of the entire body while simultaneously acquiring metabolic information about the tumor. We can correlate that metabolic information back to other anatomical and functional imaging data acquired from the MRI. The ability to do this, potentially in a single one-hour examination, is particularly attractive, both from a patient's point of view and from the standpoint of busy radiology departments with increasing demands placed on limited MRI resources.

The use of whole-body MRI as a screening tool has also been advocated [44]. As was noted earlier, the emergence of molecular medicine and personalized medicine approaches has led to extensive cancer streaming programs and identification of patients with cancer susceptibility syndromes. These patients naturally expect to have some means of radiological screening to assess for the presence of malignancy. Furthermore, they will likely require repeated long-term surveillance, since their risk of malignancy typically increases with age. The use of ultrasound as a screening tool will be limited essentially to the abdomen and would not be useful in assessing other sites of disease where malignancies often develop in patients with susceptibility syndromes (e.g., Li-Fraumeni syndrome). It is not reasonable to propose routine $\mathrm{CT}$ scanning as a means of screening these patients, particularly with increasing evidence that repeat $\mathrm{CT}$ scanning can result in an increase 
in the risk of malignant disease. Furthermore, several cancer syndromes are characterized by increases in sensitivity to the development of radiation-induced malignancy. These patients, in particular, cannot be screened by CT. The same argument applies to nuclear medicine techniques, where ionizing radiation cannot be advocated for routine screening purposes.

Whole-body MRI, therefore, has emerged as what will likely be a ubiquitous tumor screening modality in the coming decade for the vast majority of patients with cancer syndromes. Furthermore, in patients who have been successfully treated and are now free of disease, the development of systematic surveillance imaging strategies will also be necessary and a sensitive technique that does not involve additional ionizing radiation is likely to be a promising tool for the long-term surveillance of the cancer survivor populations. It will remain to be seen whether whole-body MRI will be sufficiently sensitive to serve as a routine screening modality. Finally, current limitations on the numbers of scanners in place and prohibitive costs of performing the examinations will have to be readdressed if whole-body MRI is to become the routine screening technique of the future.

\section{DCE-MRI}

Dynamic contrast-enhanced MRI (DCE-MRI) emerged in the last decade as a potential means of directly assessing the effect of anti-angiogenic agents [45]. Angiogenesis has long been recognized as a key element to the pathophysiology of tumor growth and metastasis. The ingrowth of new blood vessels is required for tumor growth and the development of molecular-targeted anti-angiogenic agents continues to be a major focus of basic research and the pharmaceutical industry. For example, agents directed against the vascular endothelial growth factor (VEGF) receptor have been developed [29]. The major antiangiogenic agents fall into two categories: agents analogous to endogenous anti-angiogenesis inhibitors (i.e. those endogenous molecules that naturally prevent tumor formation from occurring due to their inherent anti-angiogenic properties), and agents that inhibit pro-angiogenic factors such as VEGF. The potential for us to measure changes in tumor perfusion has been proposed for all of the major imaging modalities, including MRI, CT, PET and ultrasound.

During the last decade, we have learned that tumor perfusion is a complex process and cannot simply be measured by the routine contrast agents in use [45]. There are contributions of direct tumor blood flow, as well as the homeostatic mechanisms that allow blood to move in and out of the tumor interstitium. Furthermore, changes in endothelial permeability also influence the release of intravascular contrast agents into the tumor interstitium, creating challenges for use of tumor perfusion as a surrogate of treatment-related inhibition of tumor vascularity. Recent studies have evaluated whether changes in tumor vascularity induced by anti-angiogenic agents and confirmed by DCE-MRI had any correlation with ultimate treatment response [46]. The results have been less than encouraging, although it must be emphasized that the DCEMRI imaging technique itself was still an effective measure of anti-angiogenic pharmacological activity. The failure of the anti-angiogenic agent to result in a sustained tumor response and to correlate with progression free and/or overall survival must be distinguished from whether the agent possessed pharmacological activity. As we enter the next decade of imaging, it will be important to distinguish between drugs that have a pharmacological effect but do not ultimately result in improvement in patient outcome from those drugs that fail to impact patient outcome as a result of poor in vivo pharmacological activity. Indeed, it has taken nearly three decades for immunotherapeutic agents, which were touted in the 1980 s as potentially curative agents for many forms of cancer, to actually show treatment efficacy. However, it took many years of clinical trials to determine that these agents were most effective in the adjunct setting where patients had minimal residual disease, rather than in the setting where patients had bulk disease [47]. Therefore, our imaging approaches, such as DCE-MRI and other related techniques that directly assess specific targeted therapies, must be viewed as just one component of the patient response to therapy. It is likely that patients will be treated with multiple agents directed at different targets and it will be important for our imaging studies to be able to distinguish between the activity of one agent and the action of another. Therefore, what on the surface may seem to be a failure of the imaging technique to document response to therapy may, in fact, be a success in identifying a means of monitoring a particular type of pharmacological agent and distinguishing its activity from its partner drugs' activity.

\section{Fluorescent imaging}

Molecular imaging has been defined by the Commission on Molecular Imaging of the American College of Radiology as the spatially localized and temporally resolved sensing of molecular and other processes in vivo [48]. Although molecular imaging techniques, such as those in nuclear medicine and MRI spectroscopy, have been in use for many years, this terminology acknowledges the rapid growth of a new area of radiological investigation directed at developing tools to allow the specific imaging of cellular and molecular events. One of the most exciting of these molecular imaging 
techniques has been the development of fluorescent imaging strategies to evaluate malignant disease [49]. In vivo fluorescent imaging largely relies on fluorescence in the near-infrared (NIR) range of the light spectrum. These wavelengths of light penetrate biological tissues further than visible or infrared light, which are absorbed by abundant biomolecules, such as hemoglobin or water, respectively. Near-infrared imaging probes have been developed that are activated in vivo when the probes encounter tumor-specific enzymes, for example, matrix metalloproteinase. Experimental approaches using (1) near-infrared nanoprobes to selectively monitor gene transfer, and (2) the use of quantum dot targeted nano particles selectively tuned to fluoresce in specific nearinfrared spectrum wavelengths, are likely to further contribute to the expansion of NIR fluorescing nanoprobes in the imaging of cancer [8, 49].

Although studies testing the feasibility of fluorescent-based approaches to image tumors have been limited largely to the laboratory, several approaches germane to pediatric oncology are beginning to enter the clinical arena. While the depth of penetration of near-infrared light still limits this technique to structures relatively near the imaging surface, there is now potential for near-infrared fluorescent imaging probes to be used intraoperatively to provide guidance in determining tumor margins and sites of sentinel lymph node spread, and to evaluate cavities and tissue linings, such as the peritoneum, which are poorly imaged by other techniques [50]. We should imagine that this technique will be an adjunct to our existing techniques. For example, as shown in Fig. 5, a potentially metastatic lung nodule identified by $\mathrm{CT}$ was localized using CT guidance, followed by injection of methylene blue into the CT-targeted lesion. The lesion, now tattooed blue, could be readily detected on the lung in the operating room, even when the lung had been collapsed during the thoracoscopic wedge resection procedure. Frangioni and colleagues have developed near-infrared surgical microscopy and endoscopy tools to allow intraoperative and endoscopic visualization of tissues $[49,51]$. The use of tumor-targeted near-infrared fluorescent probes, therefore, will likely contribute significantly to providing tumor assessment and intraoperative surgical guidance for tumor resection, lymph node identification and targeted biopsy. In the future, we should expect the use of multiple imaging modalities, including near-infrared fluorescent probes, to provide complementary information both about tumor physiology and tumor location.

\section{Validation of new imaging technologies}

In the short term, many of these technical innovations described above are still considered experimental; however, a major effort should be focused on collecting accurate data to validate the use of these techniques clinically. For example, the current standard of care for patients with Hodgkin lymphoma is to undergo a staging CT examination and a staging FDG-PET scan. Following two cycles of chemotherapy, an early response assessment is made utilizing CT and FDG-PET imaging. As shown in Fig. 6, residual soft-tissue abnormalities are frequently present and are usually FDG negative. The use of MRI, with diffusion imaging, may be an important advance in how we image these patients, helping to distinguish an FDG-negative residual tumor from FDGnegative scar tissue. However, we will need to rigorously evaluate the use of MRI for the staging and response assessment of patients with Hodgkin lymphoma to determine whether the current sensitivity and specificity for identifying malignant disease by $\mathrm{CT}$ are matched by MRI. Once this is shown, we should be able to rapidly adapt our protocols to limit the routine use of CT scanning in this patient population, and to further encourage the use of MRI in the early diagnosis and staging, as well as follow-up surveillance imaging.

For the majority of pediatric tumors, examples such as this exist. A recent study evaluating the use of FDG-PET in patients with Wilms tumor, showed FDG accumulation in patients with Wilms tumor and demonstrated efficacy in both lesion detection and response assessment [52]. However, to incorporate FDG-PET imaging into the routine
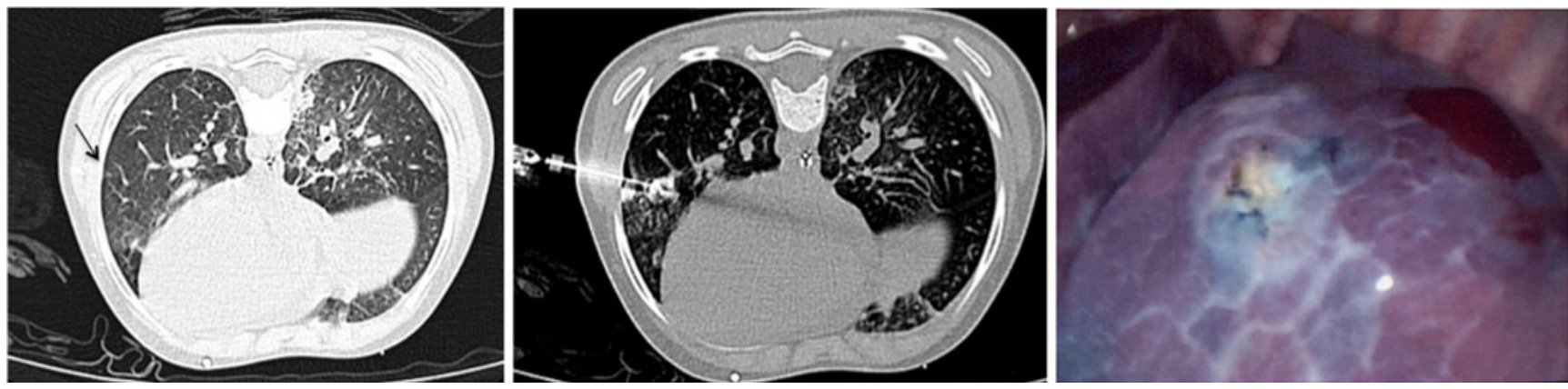

Fig. 5 Suspicious lung nodule in a child with Wilms tumor, localized by $\mathrm{CT}$ and tattooed using methylene blue. The thoracoscopic image shows localization of the lesion using the dye; however, an opportunity for substantial improvement using fluorescent agents and NIR optics exists for cases such as this. (Courtesy of Dr. Harry Padua and Dr. Christopher Weldon) 
Fig. 6 Chest and abdomen images. a Axial and coronal contrast-enhanced CT images of the chest and abdomen, respectively, show a large mediastinal mass with accompanying diffuse splenic involvement and mesenteric lymph node enlargement. The accompanying ${ }^{18} \mathrm{~F}$-FDGPET image shows diffuse FDG uptake throughout the entire mass in both the chest and abdomen. b Following two 4week cycles of therapy, there is still residual mediastinal softtissue abnormality, although it has decreased from the baseline exam (a). The spleen is now normal in size, but punctate hypodensities are still present throughout the spleen. Despite these residual CT findings, the accompanying ${ }^{18} \mathrm{~F}$-FDG-PET shows complete resolution of FDG-uptake in the mediastinal mass and in the abdomen. Reproduced with permission: [56]

\section{a}
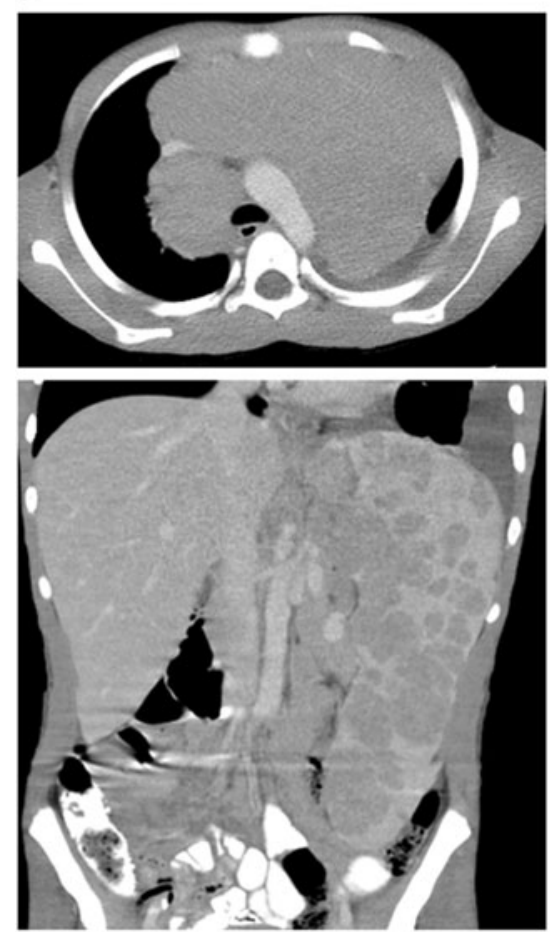

b
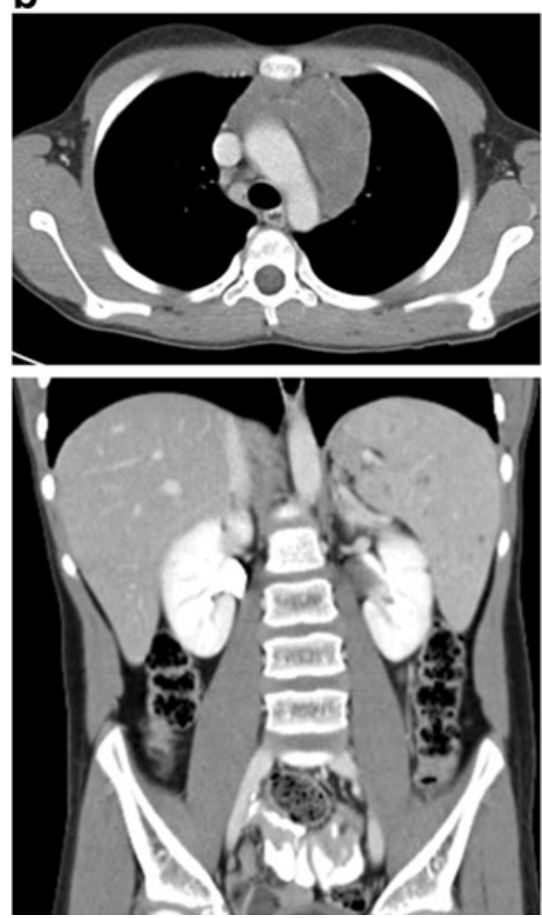
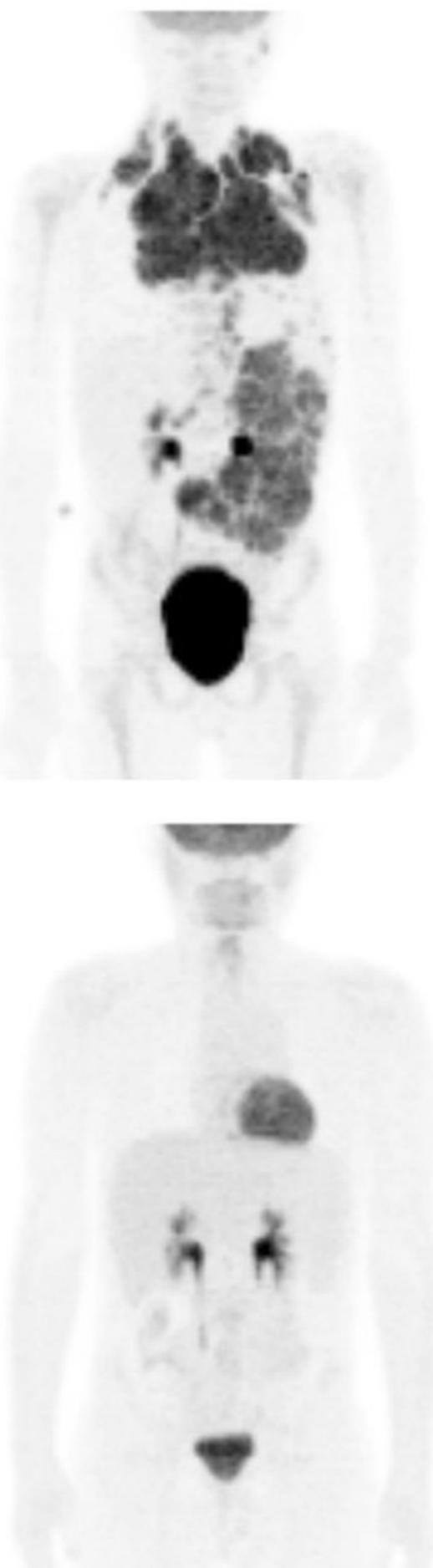

assessment of these patients, formal clinical trials will be required. Validating and comparing FDG-PET to existing techniques and correlating the imaging results with ultimate outcome data will help determine whether the added use of FDG-PET in any way influences our ability to predict outcome/early treatment relapse. Similar studies are needed for rhabdomyosarcoma, Ewing sarcoma, osteosarcoma,
non-Hodgkin lymphoma and germ cell tumors. Furthermore, in some instances, the technology may not be universally effective, as has been suggested for the use of FDG-PET in some stages of neuroblastoma [53].

An additional goal in our application and evaluation of new and innovative imaging techniques is the fundamental requirement that we extract as much information as possible 
from the imaging data we acquire. There are opportunities to review $\mathrm{CT}$ doses in an effort to further limit radiation exposure. The use of 3-D modeling techniques should be routine in every oncological planning protocol. In an era of cost containment and quality/outcome-based care initiatives, we should be making every effort to perform those studies that are most useful and most closely correlated with patient outcome. Much of what we do is dictated by routine, with new techniques added onto pre-existing examinations. In the next decade, it will essential to reassess our approach to imaging pediatric oncology patients, in an effort to reduce unnecessary scans, to increase the amount of information we extract from a given scan, to incorporate as much targeted or specific imaging as possible and to reduce unnecessary radiation exposure whenever feasible. Most of the new innovations that we can expect to enter the clinical arena have already been developed and simply await rigorous clinical testing and correlation with efficacy and outcome data in order to bring them into routine use.

\section{Multiparametric imaging of response to therapy}

The most glamorous new technology will be of little value if we cannot show that it improves patient outcome. However, in this new era of nanomedicine and personalized medicine, with targeted tumor specific imaging probes being used together with multimodality, multiplatform imaging techniques, we should not expect that any single measure of response will ultimately predict patient outcome. For the last decade, clinical trials and response assessments have been almost entirely based on standardized guidelines developed for response assessment in solid tumors, as developed by the Response Evaluation Criteria in Solid Tumors Group (RECIST). These guidelines, and recent revisions [54], still rely on sequential measurements of target lesions with extrapolation of those target measurements to overall treatment response. There are no functional criteria included in the RECIST guidelines. In the next decade, it is likely that new criteria, moving beyond RECIST, will be necessary to fully assess treatment efficacy.

Padhani and Miles have recently introduced the concept of multiparametric imaging of tumor response to therapy [55]. This concept is comparable to the multivariate analyses commonly used statistically to allow the simultaneous observation and assessment of multiple variables on a particular outcome, such as risk of developing tumor or response to therapy. The concept of multiparametric imaging of tumor response incorporates functional imaging techniques such as those described here, including FDGPET, DCE-MRI, contrast-enhanced ultrasound, and diffusion-weighted imaging techniques, among others.
Additional complementary functional PET imaging techniques are also included. This multiparametric imaging approach is then integrated together with pathological, histochemical and other clinical and laboratory data to provide what is effectively a suite of validated biomarkers. The use of image processing tools to integrate such a suite of biomarkers together should allow one to develop a reproducible, personalized, disease- and treatment-specific means of monitoring disease activity and response to therapy. The development of such a multiparametric biomarker response assessment strategy will be essential as treatments begin to evolve from risk-adapted approaches into an era of targeted response-based therapy.

\section{Conclusion}

The use of imaging in response assessment has clearly evolved. Current approaches will include a combination of both anatomical and functional imaging techniques to allow us to best predict which patients will benefit from less toxic treatment regiments, and which patients require augmented therapy. As we evolve from risk-adapted treatment strategies to guide these decisions and enter an era of responsebased therapy, the development of imaging surrogates of response to therapy should give us a specific and continuous indication of disease activity and response to therapy. Imaging allows for noninvasive biomarker development. The current challenges are to develop and validate in a rigorous controlled setting a reproducible suite of biomarkers and imaging processing tools, as well as reproducible image acquisition techniques to best accomplish these goals. It is with such an approach that the exciting technological advances delivered to us during the past decade will be incorporated into new and effective strategies for diagnosing, treating and monitoring children with cancer.

\section{References}

1. Zubrod C, Schneiderman M, Frei E et al (1960) Appraisal of methods for the study of chemotherapy of cancer in man: comparative therapeutic trial of nitrogen mustard and triethylene thiophosphoramide. J Chronic Dis 11:7-33

2. Adamson PC (2009) Imaging in early phase childhood cancer trials. Pediatr Radiol 39(Suppl 1):S38-S41

3. Reaman GH (2009) What, why, and when we image: considerations for diagnostic imaging and clinical research in the Children's Oncology Group. Pediatr Radiol 39(Suppl 1):S42S45

4. Shankar LK, Van den Abbeele A, Yap J et al (2009) Considerations for the use of imaging tools for phase II treatment trials in oncology. Clin Cancer Res 15:1891-1897

5. Miller AB, Hoogstraten B, Staquet M et al (1981) Reporting results of cancer treatment. Cancer 47:207-214 
6. Kim BY, Rutka JT, Chan WC (2010) Nanomedicine. N Engl J Med 363:2434-2443

7. LaRocque J, Bharali DJ, Mousa SA (2009) Cancer detection and treatment: the role of nanomedicines. Mol Biotechnol 42:358-366

8. Portney NG, Ozkan M (2006) Nano-oncology: drug delivery, imaging, and sensing. Anal Bioanal Chem 384:620-630

9. Willmann JK, van Bruggen N, Dinkelborg LM et al (2008) Molecular imaging in drug development. Nat Rev Drug Discov 7:591-607

10. Petak I, Schwab R, Orfi L et al (2010) Integrating molecular diagnostics into anticancer drug discovery. Nat Rev Drug Discov 9:523-535

11. Schilsky RL (2010) Personalized medicine in oncology: the future is now. Nat Rev Drug Discov 9:363-366

12. Aebersold R, Auffray C, Baney E et al (2009) Report on EU-USA workshop: how systems biology can advance cancer research (27 October 2008). Mol Oncol 3:9-17

13. Therasse P, Arbuck SG, Eisenhauer EA et al (2000) New guidelines to evaluate the response to treatment in solid tumors. European Organization for Research and Treatment of Cancer, National Cancer Institute of the United States, National Cancer Institute of Canada. J Natl Cancer Inst 92:205-216

14. Suzuki C, Jacobsson H, Hatschek T et al (2008) Radiologic measurements of tumor response to treatment: practical approaches and limitations. Radiographics 28:329-344

15. Barnacle AM, McHugh K (2006) Limitations with the response evaluation criteria in solid tumors (RECIST) guidance in disseminated pediatric malignancy. Pediatr Blood Cancer $46: 127-134$

16. Desar IM, van Herpen CM, van Laarhoven HW et al (2009) Beyond RECIST: molecular and functional imaging techniques for evaluation of response to targeted therapy. Cancer Treat Rev 35:309-321

17. Portwine C, Marriott C, Barr RD (2010) PET imaging for pediatric oncology: an assessment of the evidence. Pediatr Blood Cancer 55:1048-1061

18. McCarville MB (2009) PET-CT imaging in pediatric oncology. Cancer Imaging 9:35-43

19. Franzius C (2010) FDG-PET/CT in pediatric solid tumors. Q J Nucl Med Mol Imaging 54:401-410

20. Kleis M, Daldrup-Link H, Matthay K et al (2009) Diagnostic value of PET/CT for the staging and restaging of pediatric tumors. Eur J Nucl Med Mol Imaging 36:23-36

21. Furth C, Steffen IG, Amthauer H et al (2009) Early and late therapy response assessment with [18F]fluorodeoxyglucose positron emission tomography in pediatric Hodgkin's lymphoma: analysis of a prospective multicenter trial. J Clin Oncol 27:4385-4391

22. Gallamini A, Hutchings M, Avigdor A et al (2008) Early interim PET scan in Hodgkin lymphoma: where do we stand? Leuk Lymphoma 49:659-662

23. Hawkins DS, Conrad EU 3rd, Butrynski JE et al (2009) [F-18]fluorodeoxy-D-glucose-positron emission tomography response is associated with outcome for extremity osteosarcoma in children and young adults. Cancer 115:3519-3525

24. Hawkins DS, Schuetze SM, Butrynski JE et al (2005) [18F] Fluorodeoxyglucose positron emission tomography predicts outcome for Ewing sarcoma family of tumors. J Clin Oncol 23:8828-8834

25. Weckesser M (2009) Molecular imaging with positron emission tomography in paediatric oncology-FDG and beyond. Pediatr Radiol 39(Suppl 3):450-455

26. Voss SD, Smith SV, DiBartolo N et al (2007) Positron emission tomography (PET) imaging of neuroblastoma and melanoma with 64Cu-SarAr immunoconjugates. Proc Natl Acad Sci USA 104:17489-17493
27. Mach RH, Dehdashti F, Wheeler KT (2009) PET radiotracers for imaging the proliferative status of solid tumors. PET Clin $4: 1-15$

28. Malempati S, Weigel B, Ingle AM et al (2009) A phase I trial and pharmacokinetic study of IMC-A12 in pediatric patients with relapsed/refractory solid tumors: A Children's Oncology Group Phase I Consortium study. J Clin Oncol 27:15s (suppl; abstr 10013)

29. Heneweer C, Grimm J (2011) Clinical applications in molecular imaging. Pediatr Radiol 41:199-207

30. Pichler BJ, Kolb A, Nagele T et al (2010) PET/MRI: paving the way for the next generation of clinical multimodality imaging applications. J Nucl Med 51:333-336

31. Judenhofer MS, Wehrl HF, Newport DF et al (2008) Simultaneous PET-MRI: a new approach for functional and morphological imaging. Nat Med 14:459-465

32. Robbins E (2008) Radiation risks from imaging studies in children with cancer. Pediatr Blood Cancer 51:453-457

33. Alessio AM, Kinahan PE, Manchanda V et al (2009) Weightbased, low-dose pediatric whole-body PET/CT protocols. J Nucl Med 50:1570-1577

34. Sauter AW, Wehrl HF, Kolb A et al (2010) Combined PET/MRI: one step further in multimodality imaging. Trends Mol Med 16:508-515

35. Wehrl HF, Sauter AW, Judenhofer MS et al (2010) Combined PET/MR imaging-technology and applications. Technol Cancer Res Treat 9:5-20

36. Padhani AR, Liu G, Koh DM et al (2009) Diffusion-weighted magnetic resonance imaging as a cancer biomarker: consensus and recommendations. Neoplasia 11:102-125

37. Afaq A, Andreou A, Koh DM (2010) Diffusion-weighted magnetic resonance imaging for tumour response assessment: why, when and how? Cancer Imaging 10 Spec no A:S179S188

38. Koh DM, Collins DJ (2007) Diffusion-weighted MRI in the body: applications and challenges in oncology. AJR 188:16221635

39. Murphy P, Koh DM (2010) Imaging in clinical trials. Cancer Imaging 10 Spec no A:S74-S82

40. Humphries PD, Sebire NJ, Siegel MJ et al (2007) Tumors in pediatric patients at diffusion-weighted MR imaging: apparent diffusion coefficient and tumor cellularity. Radiology 245:848-854

41. Kwee TC, Takahara T, Ochiai R et al (2009) Whole-body diffusion-weighted magnetic resonance imaging. Eur $\mathrm{J}$ Radiol 70:409-417

42. Darge K, Jaramillo D, Siegel MJ (2008) Whole-body MRI in children: current status and future applications. Eur J Radiol 68:289-298

43. Krohmer S, Sorge I, Krausse A et al (2010) Whole-body MRI for primary evaluation of malignant disease in children. Eur J Radiol $74: 256-261$

44. Ladd SC (2009) Whole-body MRI as a screening tool? Eur J Radiol 70:452-462

45. Barrett T, Brechbiel M, Bernardo M et al (2007) MRI of tumor angiogenesis. J Magn Reson Imaging 26:235-249

46. Hahn OM, Yang C, Medved M et al (2008) Dynamic contrastenhanced magnetic resonance imaging pharmacodynamic biomarker study of sorafenib in metastatic renal carcinoma. J Clin Oncol 26:4572-4578

47. Shusterman S, London WB, Gillies SD et al (2010) Antitumor activity of hu14.18-IL2 in patients with relapsed/refractory neuroblastoma: a Children's Oncology Group (COG) phase II study. J Clin Oncol 28:4969-4975

48. Miller JC, Thrall JH (2004) Clinical molecular imaging. J Am Coll Radiol 1:4-23

49. Frangioni JV (2008) New technologies for human cancer imaging. J Clin Oncol 26:4012-4021 
50. Choi HS, Liu W, Liu F et al (2010) Design considerations for tumour-targeted nanoparticles. Nat Nanotechnol 5:42-47

51. Troyan SL, Kianzad V, Gibbs-Strauss SL et al (2009) The FLARE intraoperative near-infrared fluorescence imaging system: a firstin-human clinical trial in breast cancer sentinel lymph node mapping. Ann Surg Oncol 16:2943-2952

52. Begent J, Sebire NJ, Levitt G et al (2011) Pilot study of F(18)fluorodeoxyglucose positron emission tomography/computerised tomography in Wilms' tumour: correlation with conventional imaging, pathology and immunohistochemistry. Eur J Cancer 47:389-396
53. Sharp SE, Shulkin BL, Gelfand MJ et al (2009) 123I-MIBG scintigraphy and 18F-FDG PET in neuroblastoma. J Nucl Med 50:1237-1243

54. Eisenhauer EA, Therasse P, Bogaerts J et al (2009) New response evaluation criteria in solid tumours: revised RECIST guideline (version 1.1). Eur J Cancer 45:228-247

55. Padhani AR, Miles KA (2010) Multiparametric imaging of tumor response to therapy. Radiology 256:348-364

56. Voss SD (2010) Imaging and response assessment in Hodgkin lymphoma. Am Soc Clin Oncol Ed Book, 397-407 